\title{
POSTERS
}

\section{Expansion Analyses on Planetary Nebulae}

\author{
S. Tamura ${ }^{1}$, Y. Yadoumaru ${ }^{2}$, K.M. Shibata ${ }^{2}$ and A. Tajitsu ${ }^{1}$ \\ ${ }^{1}$ Astronomical Institute, Tohoku University; ${ }^{2}$ National Astronomical Observatory, Mitaka
}

Since planetary nebulae(PNe) are defined as the expanding ionized gas surrounding hot central stars, the most important and fundamental subject is to study expansion characteristics. What and how can we determine a typical expansion velocity of individual nebula? In spite of great success of Perek and Kohoutek Catalog(1967) and a recent StrasbourgESO Catalogue(1992), PNe researchers have suffered from the lack of sufficiently large samples of expansion measurements on various type of PNe.

We have obtained expansion velocities $\left(2 \mathrm{~V}_{\text {exp }}\right)$ of 76 nebulae by using the emissionlines of $\mathrm{H}_{\alpha}$, [OIII]5007, and HeII4686 within the accuracy of $8 \mathrm{~km} / \mathrm{sec}\left(\right.$ for $\mathrm{H}_{\alpha}$ ) or less. These velocities are defined by the difference between two peak velocities in case of well developed nebulae $\left(2 \mathrm{~V}_{\text {exp }}=\left|\mathrm{V}_{p 1}-\mathrm{V}_{p 2}\right|\right)$ or the FWHM of undeveloped ones in each line $\left(2 \mathrm{~V}_{\text {exp }}=\left(\mathrm{V}_{F W H M}^{2}-\mathrm{V}_{t h}^{2}-\mathrm{V}_{\text {inst }}^{2}\right)^{1 / 2}\right.$, where $\mathrm{V}_{t h}$ is the thermal velocity of ion, and $\mathrm{V}_{\text {inst }}$ is the velocity derived from the instrumental profile. We must pay our attention to the position angles of slit projected to the nebula if we would try to compare the velocities of different ions and emission lines. We examine the characteristics of nebular expansion based upon these velocities in comparison with distance free parameters as well as distance dependent observed quantities. The distances to PNe are determined by our own work (Tajitsu and Tamura, poster of this symposium, I-13). 76 nebulae can be classified into two groups, low excitation and high excitation nebulae.

There exists the lower limit of expansion velocity in the [OIII]5007 line which is around $10 \mathrm{~km} / \mathrm{sec}$ in case of $2 \mathrm{~V}_{e x p}=\left|\mathrm{V}_{p 1}-\mathrm{V}_{p 2}\right|$, and may be due to the instrumental effect. We can supplement smaller values with the expansion velocities based on $\mathrm{V}_{F W H M}$ under the assumption of some empirical relation to $\left|\mathrm{V}_{p 1}-\mathrm{V}_{p 2}\right|$.

When we adopt the electron density as a distance free parameter, we can see the relationship between $2 \mathrm{~V}_{\text {exp }}$ and the density which indicates the stationary flow among sample nebulae as a whole as suggested previously by Tamura and Shibata(1990).

It is conspicuous for the linear inclination of $2 \mathrm{~V}_{\text {exp }}$ up to $50 \mathrm{~km} / \mathrm{sec}$ from $10 \mathrm{~km} / \mathrm{sec}$ versus radii corresponding to about maximum $0.2 \mathrm{pc}$ and less than $0.05 \mathrm{pc}$. In this part almost all of samples are occupied by high excitation nebulae. Beyond the linear inclination part $2 \mathrm{~V}_{\text {exp }}$ shows scattering, but rather mild increase depending on radii.

\section{REFERENCES}

Acker, A., Ochsenbein, F., Stenholm, B., Tylenda, R., Marcout, J., Schohn, C., 1992,

ESO, München.

Perek, L. and Kohoutek, L. 1967, Czechslovak Academy of Science, Prague.

Tamura, S. and Shibata, K.M., 1990, PASP, 102, 1301. 